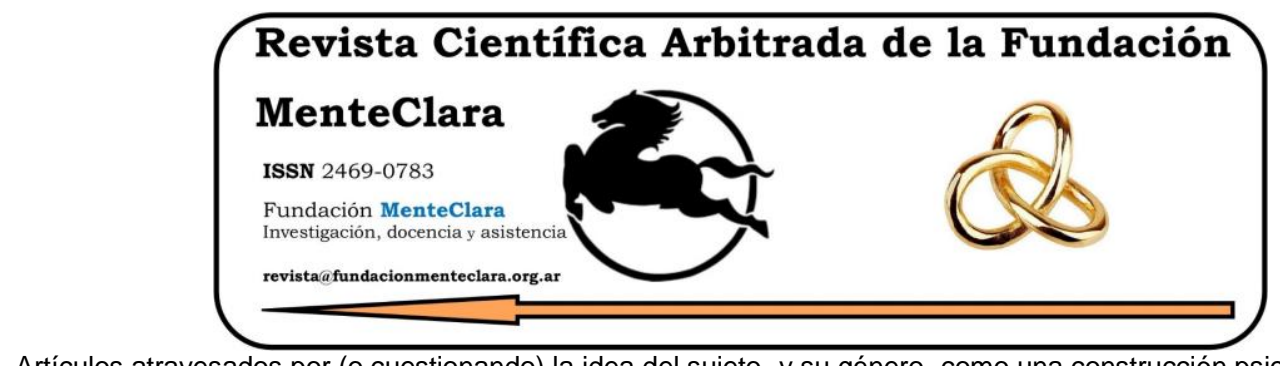

Artículos atravesados por (o cuestionando) la idea del sujeto -y su género- como una construcción psicobiológica de la cultura. Articles driven by (or questioning) the idea of the subject -and their gender- as a cultural psychobiological construction. Vol. 5 (2020), enero-diciembre ISSN 2469-0783 https://datahub.io/dataset/2020-5-e199

\title{
EL OPTIMISMO Y SU RELACIÓN CON EL BIENESTAR PSICOLÓGICO
}

\section{OPTIMISM AND IT’S RELATION WITH PSYCHOLOGICAL WELL-BEING}

Camila Ferrer Camila.ferrer@uflouniversidad.edu Facultad de Psicología y Ciencias Sociales, Universidad de Flores, Argentina

Cómo citar este artículo / Citation: Ferrer Camila, N. (2020). El optimismo y su relación con el bienestar psicológico. Revista Científica Arbitrada de la Fundación MenteClara, Vol.5 (199). DOI: https://doi.org/10.32351/rca.v5.199

Copyright: (C) 2020 RCAFMC. Este artículo de acceso abierto es distribuido bajo los términos de la licencia Creative Commons Attribution 4.0 International License (CC BY 4.0). Recibido: 29/11/2020. Aceptado: 07/12/2020 Publicación online: 10/12/2020

Conflicto de intereses: Ninguno que declarar.

\section{Resumen}

Los constructos optimismo y bienestar psicológico han sido estudiados con mayor dedicación durante los últimos años, desde el enfoque de la psicología positiva. El objetivo del presente trabajo es indagar la relación existente entre ambas variables mediante una revisión sistemática de la literatura previa, sintetizando de tal modo el estado del arte del mencionado vínculo. A partir de la revisión se concluye que el optimismo se asocia fuertemente y de manera positiva al bienestar psicológico. Además, el primero constituye un predictor del segundo. Sin embargo, se sugiere continuar indagando sobre el vínculo entre estos dos constructos para conocer con mayor certeza la naturaleza del mismo.

\footnotetext{
Abstract

Optimism and psychological well-being have been studied with greater dedication in recent years, from the perspective of positive psychology. The objective of this work is to investigate the relationship between both variables by means of a systematic review of the previous literature, thus synthesizing the state of the art of the aforementioned link. From the review, it is concluded that optimism is strongly and positively
} 
associated with psychological well-being. Furthermore, the former is a predictor of the latter. However, it is suggested to continue investigating the link between these two constructs in order to know its nature with greater certainty.

Palabras Claves: Optimismo; bienestar psicológico

Keywords: optimism; psychological well-being 


\section{Introducción}

Históricamente, la psicología se ha centrado en reducir el dolor y el sufrimiento, así como en las carencias de capacidades individuales o colectivas. La psicología de la salud aporta una visión que podría considerarse dejada de lado por el foco tradicional; la que vislumbra elementos positivos y ofrece un estudio enfocado al bienestar humano. La creciente mirada positiva se explicitó en la definición de "salud" otorgada por la Organización Mundial de la Salud en 1948, que rompió con la conceptualización de la misma como la mera ausencia de enfermedad. En ella, salud es concebida como un completo estado de bienestar tanto físico, como mental y social (OMS, 1948), considerando las tres esferas, biológica, psicológica y social, del ser humano.

Actualmente continúan realizándose estudios que dan cuenta de la importancia y de los vínculos que presentan entre sí las distintas variables psicológicas positivas. El optimismo es una de las que más presencia ha adquirido en los últimos años. Otra variable de gran importancia la constituye el bienestar psicológico; componente primordial de la salud, como previamente se mencionó.

El propósito del presente trabajo será aportar una sintesis sobre el estado del arte de la relación entre los constructos mencionados, a partir de una revisión sistemática de la literatura previa llevada a cabo en bases de datos científicas.

\section{Optimismo}

El optimismo ha sido definido de formas diversas. Principalmente, es entendido desde dos perspectivas teóricas diferentes pero complementarias. 
Por un lado, como estilo inferencial -o explicativo- positivo (Seligman et al., 1978) según el cual los eventos negativos son considerados inestables -permanencia-, específicos -amplitud/ubicuidad-y debidos a causas externas -causalidad-.

Según investigaciones previas, un estilo explicativo optimista es predictor de una mejor salud y menor vulnerabilidad a las enfermedades físicas, en contraparte al estilo explicativo pesimista, vinculado con más problemas de salud y mortalidad (Peterson et al., 1988), así como también se encontró positivamente relacionado con resoluciones racionales y positivas de problemas (Sarmiento \& Fritz, 2018) y es protector y combatiente de sintomatología depresiva (Ojeda et al., 2019). De acuerdo a un estudio longitudinal cuya muestra constaba de estudiantes universitarios, el estilo inferencial optimista supone una menor propensión a padecer ansiedad, afecto negativo e informe de sintomas somáticos, así como una menor depresión (Pérez-Cacho, 2016), concordando así, con estudios previos.

Por otro lado, encontramos el optimismo disposicional. El mismo es definido por Carver y Scheier (2014) como una construcción cognitiva referida a expectativas positivas respecto al futuro, encontrándose relacionado a la motivación y mayor esfuerzo.

E1 optimismo disposicional disminuye los niveles de todas las dimensiones del burnout, según un estudio llevado a cabo sobre una población deportista (Pérez, 2017). También es predictor de la resiliencia en estudiantes universitarios, de acuerdo a una investigación realizada por Gómez-Molinero et al. (2018) y se encuentra relacionado positivamente a la satisfacción con la vida en estudiantes universitarios italianos de ambos sexos (Cabras \& Mondo, 2017). Además, se encuentra vinculado de forma positiva con la autoestima y la resiliencia psicológica, y de forma negativa con los síntomas depresivos, según un estudio 
realizado por Kapikiran y Acun-Kapikiran (2016) con una muestra de estudiantes universitarios. Asimismo, un estudio llevado a cabo sobre estudiantes de 4to año de la carrera de Psicología (Vera, 2016) afirma que el optimismo disposicional se relaciona negativamente con la aparición de sintomas somáticos, ansiedad, depresión y afecto negativo.

\section{Optimismo y Bienestar Psicológico}

El bienestar constituye un término amplio cuya definición ha sido debatida y abarcada en el marco de múltiples conceptualizaciones y diversos fundamentos teóricos. El mismo puede entenderse como un estado de la persona cuyas condiciones físicas y mentales le proporcionan un sentimiento de satisfacción y tranquilidad (Bermúdez, 2017).

Con el tiempo, el estudio del bienestar ha marcado una diferenciación; por un lado el bienestar subjetivo, y por el otro, el bienestar psicológico. Dicha distinción es también abordada desde las concepciones de "hedonismo" y "eudaimonia" respectivamente (Kardas et al., 2019). E1 primero, por su parte, se encuentra relacionado al afecto negativo y positivo, vinculado a las dimensiones emocionales del individuo, y a la satisfacción con la vida. El segundo se vincula a la autoaceptación, refiriendo a una actitud positiva hacia uno mismo y hacia los diferentes aspectos de la realidad y del pasado (Bermúdez, 2017).

El término bienestar psicológico es caracterizado por ser multidimensional y, como sostienen Barrientos et al. (2017), puede ser definido como el crecimiento personal y las capacidades de un individuo, a partir de las cuales éste muestra indicadores de un funcionamiento positivo. Constituye, afirman los mismos, una experiencia personal que se construye a lo largo del tiempo con el desarrollo psicológico del sujeto 
y con la capacidad de relacionarse positivamente con sus experiencias, teniendo en cuenta la incorporación activa de medidas positivas y no solamente la ausencia de aspectos negativos vivenciados.

Diversos autores han otorgado múltiples definiciones del bienestar psicológico. Por su parte, Carol Ryff (1989, citada en Freire et al., 2017), ideó un modelo de bienestar psicológico -o eudaimónico- conformado por seis dimensiones: autoaceptación, autonomía, dominio del entorno, relaciones positivas con otros, propósito en la vida y crecimiento personal. Barrientos et al. (2017) afirman que el bienestar psicológico puede medirse a través de los componentes que posee: afectos y cognición, siendo éstos analizados en diferentes contextos, ya sea trabajo, estudio, familia.

Existen numerosos estudios que abordan ambos constructos, optimismo y bienestar psicológico, y que dan cuenta la relación que estos dos presentan.

Por su parte, Cnen et al. (2020) abordan al bienestar psicológico a partir de cuatro de las dimensiones planteadas por Ryff; autonomía, propósito en la vida, relaciones positivas con los demás y crecimiento personal. Los mismos, partiendo de un estudio que llevaron a cabo en el cual participaron 322 deportistas estudiantes universitarios, concluyeron que el optimismo entendido como expectativas positivas para el futuro y medido con la escala LOT-R -Life Orientation Test Revised y todas las dimensiones del bienestar psicológico medido con la Psychologycal well Being Scale, además del apoyo social brindado por los entrenadores, presentan altas correlaciones positivas; el optimismo apareció como mediador en la relación entre el apoyo social y el bienestar psicológico. El pesimismo, por otro lado, se asoció negativamente al bienestar psicológico. 
De igual manera, Jahanara (2017) quien estudió la relación entre el optimismo, la esperanza y la salud mental -bienestar psicológico y distress psicológico-, afirma que los primeros dos influyen en el tercero, encontrándose el optimismo asociado positivamente al bienestar psicológico al igual que la esperanza y negativamente con el distress psicológico. Por ende, sostiene, el optimismo tiene influencia en la salud mental. Al igual que el estudio anterior, el optimismo fue evaluado con la escala LOT-R, mientras que la salud mental, abarcativa del bienestar psicológico fue medida con el MHI -Mental Health Inventory-.

Otra investigación que abordó la temática optimismo-bienestar psicológico, además de incluir la vitalidad, el pensamiento positivo y la regulación emocional, es la realizada por Nejad et al. (2019). En ella, el bienestar psicológico es concebido desde las mismas seis dimensiones de Ryff expuestas previamente, mientras que el optimismo es entendido desde el estilo explicativo positivo de Seligman ya mencionado. Los resultados arrojados indican que el optimismo tiene una fuerte y positiva relación con el bienestar psicológico y con la regulación emocional. De forma coincidente, Krok y Telka (2019) concluyeron que el optimismo y el tener propósito de vida se relacionan significativamente con el bienestar psicológico y con el subjetivo, constituyendo el optimismo un mediador entre el propósito de vida y el bienestar psicológico. Dichos resultados fueron obtenidos tras un estudio realizado sobre 205 adolescentes, en el cual el optimismo disposicional fue medido con la escala Life Orientation Test - Revised, y el bienestar psicológico, enfocado al crecimiento y desarrollo personal a la par del tener metas significativas y el superar desafios en la vida, fue evaluado con el Psychological Well Being Scale.

La investigación llevada a cabo por Adekunle (2017) también respalda la relación positiva entre optimismo y bienestar psicológico; el estudio se 
realizó en una ciudad de Nigeria y su muestra constaba de 276 policías. Se descubrió que la inteligencia emocional y el optimismo disposicional se vinculan positivamente con el bienestar psicológico, teniendo además un carácter predictivo del mismo. Asimismo, Wani (2018), en su estudio sobre la depresión, el optimismo y el bienestar psicológico en 100 adolescentes hombres y mujeres, encontró que aquellos que presentaban altos niveles de optimismo informaban mayores niveles de bienestar psicológico y menores niveles de depresión que aquellos que presentaban niveles más pesimistas.

Como se ha expuesto, parece haber cierta unanimidad con respecto a la relación positiva que presentan el optimismo y el bienestar psicológico dentro de la comunidad científica, puesto que los estudios empíricos realizados así lo sugieren.

\section{Optimismo como predictor del Bienestar Psicológico}

Existe una diferencia entre establecer un mero vínculo entre dos variables y establecer una relación predictiva entre ellas. Es posible identificar estudios varios que afirman que el optimismo predice de forma positiva al bienestar psicológico.

Un interesante resultado obtenido a partir de una investigación llevada a cabo por Moreno y Marrero (2015) en una muestra conformada por 1403 adultos, reza que el optimismo, concebido una vez más como expectativas positivas para el futuro y medido con la escala LOT-R, junto con la autoestima, predicen entre un 16\% y un 55\% de la varianza de los seis mencionados indicadores del bienestar psicológico medido con la Psychological Well-Being Scale. El mismo también reveló la existencia de una diferencia de género; los hombres puntuaron más alto en ambos predictores que las mujeres. Otro porcentaje no muy lejano es el brindado 
por Hoseini et al. (2016), que expone que el optimismo, esta vez interpretado a partir de la definición otorgada por Seligman anteriormente mencionada, como estilo explicativo optimista y la persistencia son fuertes predictores del bienestar psicológico, explicando un $29.07 \%$ del mismo. Su estudio contaba con una muestra conformada por un conjunto de enfermeras. Patki (2016) también habla de porcentajes; según su investigación sobre el optimismo, el apoyo social percibido y el bienestar psicológico en estudiantes, el optimismo nuevamente entendido como expectativas positivas para el futuro y medido con la escala LOT-R- predice un $18 \%$ de la varianza del bienestar psicológico medido con la escala Psychological General Well-Being Index, relacionándose fuerte y positivamente uno con otro.

Otras investigaciones han llegado a las mismas conclusiones; un estudio realizado por Ramnath y Singh (2019) con una muestra de 600 jóvenes adultos -300 hombres y 300 mujeres-, en el que el bienestar psicológico es entendido como un componente primordial de la salud física y mental, sostiene que el optimismo medido con la escala LOT-R Life Orientation Test - Revised y entendido también, desde la concepción mencionada de Carver y Sheier (2014) sobre expectativas positivas para el futuro, no solo se relaciona positivamente con el bienestar psicológico, sino también que predice al mismo -de igual manera la esperanza-. Estos resultados coinciden con los arrojados por Chamua y Sankar (2018); los mismos llevaron a cabo un estudio sobre estudiantes de un post grado, indagando acerca de la resiliencia, la autoestima y optimismo aprendido -el tener expectativas positivas para el futuro a pesar de los inconvenientes que presenta la vida-, como predictores del bienestar psicológico comprendido desde las seis dimensiones planteadas por Ryff. Llegaron a dos conclusiones: la totalidad de las variables mencionadas se relacionan positivamente entre sí, y el optimismo aprendido junto con 
la resiliencia y la autoestima predicen significativa y positivamente al bienestar psicológico.

Es mayor la cantidad de estudios cuyos resultados apuntan a que el optimismo predice el bienestar psicológico que los que sugieren lo contrario. Aun así, investigaciones como la llevada a cabo por Farhadian y Moradi (2020) en una muestra conformada por estudiantes, concluyen que el optimismo -interpretado y medido de igual manera que los estudios anteriormente citados-, si bien se relaciona de manera positiva, no predice significativamente el bienestar psicológico medido a través de las seis dimensiones de Ryff con el cuestionario de Ryff, a diferencia de, por ejemplo, el bienestar espiritual.

\section{Conclusiones}

De la presente investigación se concluye que existe una relación considerablemente significativa y positiva entre los constructos optimismo y bienestar psicológico -siendo el primero un componente de gran importancia del segundo-. Dicha relación se ve reflejada en el conjunto de resultados de múltiples estudios empíricos que han sido abordados en la revisión sistemática de la literatura previa llevada a cabo.

Como se desarrolló, si bien existe cierta discrepancia con respecto a ello, el optimismo parece constituir una variable predictora del bienestar psicológico, como apuntan numerosos estudios. A su causa, se sugiere estimular el optimismo por medio de diversas intervenciones, en pos de alcanzar mayores niveles de bienestar psicológico en toda población en general.

En la gran mayoría de los estudios remitidos al tema, el optimismo es considerado desde las definiciones previamente desarrolladas de Carver y Scheier (2014) y Seligman et al. (1978), que rezan sobre el optimismo 
disposicional y el estilo explicativo positivo respectivamente. También es recalcable que la escala mayormente utilizada para medir esta variable es la Life Orientation Test - Revised (propuesta por Scheier et al., 1994). La misma ha demostrado poseer validez y confiabilidad (GustemsCarnicer et al., 2017). En lo que respecta al bienestar psicológico, es generalmente concebido desde el modelo previamente mencionado de Ryff (1989) y la Psychological Well-Being Sacale es la forma más utilizada para su evaluación. Este constituye un punto importante, ya que al haber determinadas conceptualizaciones y escalas mayormente establecidas, se reduce la posibilidad de discrepancias debidas a una diversidad de concepciones que se puedan llegar a tener de los constructos o a diferentes formas en que los mismos son evaluados.

\section{Discusión}

Merece ser mencionado que la investigación en cuestión es acotada, por lo cual sería optimo agregar otros trabajos para lograr una comprensión aún más profunda sobre el vínculo de las mencionadas variables.

Asimismo, en la revisión no se contemplaron de forma específica las discrepancias existentes con respecto a las diferencias de género, edad o clase social que podrian alterar tanto a la relación planteada como a cada constructo en particular.

Más allá de las limitaciones que presenta, se considera que la presente investigación aporta de forma precisa y clara una síntesis del estado del arte del vínculo existente entre el optimismo y el bienestar psicológico, teniendo el mismo una gran relevancia en el ámbito psicológico, particularmente dentro del marco de la joven rama de la psicología positiva. 


\section{Referencias}

Abramson, L., Seligman, M., \& Teasdale, J. (1978). Learned helplessness in humans: Critique and reformulation. Revista de Psicologia Anormal, 87 (1), 49-74. https://doi.org/10.1037/0021-843X.87.1.49

Adekunle, O. S. (2017). Impact of Emotional Intelligence, Dispositional Optimism and Emotional Exhaustion on the Psychological Well-Being of Police Officers in Ibadan, Oyo State, Nigeria. International Journal of Innovation and Applied Studies, 19 (3), 717-723

Caballano Pérez-Cacho, T. (2016). Estilos atribucionales optimistas-pesimistas y su relación con la ansiedad, depresión, afectividad y sintomas somáticos. (Tesis de fin de grado, Universidad de Jaen). Repositorio institucional de la Universidad de Jaen. https://hdl.handle.net/10953.1/3412

Cabras, C., \& Mondo, M. (2017). Coping strategies, optimism, and life satisfaction among first-year university students in Italy: gender and age differences. Higher Education, 75 (4), 643-654. https://doi.org/10.1007/s10734-017-0161-x

Carver, S. C., \& Scheier, M. S. (2014). Dispositional optimism. Trends in Cognitive Sciences, 18 (6), 293-299. https://doi.org/10.1016/j.tics.2014.02.003

Chamuah, A., \& Sankar, R. (2018). Resilience, learned optimism and self-esteem as predictors of psychological well - being among university students. International Journal of Creative and Innovating Research in all Studies, 1 (5), 1-4.

Cnen, T. W., Chiu, Y. C., \& Hsu, Y. (2020). Perception of social support provided by coaches, optimism/pessimism, and psychological well-being: Gender differences and mediating effect models. International Journal of Sports Science \& Coaching, 15 (3), 112-123. https://doi.org/10.1177\%2F1747954120968649

Farhadian, F., \& Moradi, A. (2020). The contribution of spiritual health, optimism and happiness in prediction of psychological well-being among students. Knowledge \& Research in Applied Psychology, 21 (2), 93-104.

Freire, C., Ferradás, M., Núñez, J. C., \& Valle, A. (2017). Estructura factorial de las Escalas de Bienestar Psicológico de Ryff en estudiantes universitarios. European Journal of Education and Psychology, 10 (1), 1-8. https://doi.org/10.1016/i.ejeps.2016.10.001

García Bermúdez, A. G. (2017). Relación entre dependencia, bienestar psicológico y subjetivo en personas mayores. (Tesis de fin de grado, Universidad de Jaen). Repositorio institucional de la Universidad de Jaen. https://hdl.handle.net/10953.1/5978 
Gómez-Molinero, R., Zayas, A., Ruíz-González, P., \& Guil, R. (2018). Optimism and Resilience among university students. International Journal of Developmental and Educational Psychology, 1 (1), 1-8.

Gustems-Carnicer, J., Calderón, C., \& Santacana, M. F. (2017). Psychometric properties of the Life Orientation Test (LOT-R) and its relationship with psychological well-being and academic progress in college students. Revista Latinoamericana de Psicología, 49 (1), 1927. https://doi.org/10.1016/j.rlp.2016.05.001

Hoseini, Z. S., Habibmazaheri, N., \& Rahiminasab, M. (2016). The Relationships of Rational Thinking, Optimism, Persistence and Psychological Wellbeing in Nurses of Kermanshah Hospitals. Iranian Journal of Nursing Research, 11 (4), 49-56. http://dx.doi.org/10.21859/ijnr-110406

Jahanara, M. (2017). Optimism, Hope and Mental Health: Optimism, Hope, Psychological Well-Being and Psychological Distress among Students, University of Pune, India. International Journal of Medical, Health, Biomedical, Bioengineering and Pharmaceutical Engineering, 11 (12), 441-444.

Kapikiran, S., \& Acun-Kapikiran, N. (2016). Optimism and Psychological Resilience in relation to Depressive Symptoms in University Students: Examining the Mediating Role of Self-Esteem. Educational Sciences: Theory \& Practice, 16 (6), 2087-2110. https://doi.org/10.12738/estp.2016.6.0107

Kardas, F., Cam, Z., Eskisu, M., \& Gelibolu, S. (2019). Gratitude, Hope, Optimism and Life Satisfaction as Predictors of Psychological Well-Being. Eurasian Journal of Educational Research, 82 (1), 81-99. https://10.14689/ejer.2019.82.5

Krok, D., \& Telka, E. (2019). Optimism mediates the relationships between meaning in life and subjective and psychological well-being among late adolescents. Current Issues in Personality Psychology, 7 (1), 32-42. https://doi.org/10.5114/cipp.2018.79960

Mendoza-Vera, M. J. (2016). Relación del Optimismo Disposicional con la Ansiedad, Depresión, Afectividad y Síntomas Somáticos. (Tesis de fin de grado, Universidad de Jaen). Repositorio institucional de la Universidad de Jaen. https://hdl.handle.net/10953.1/3541

Moreno, Y., \& Marrero, R. J. (2015). Optimismo y Autoestima como predictores de Bienestar Personal: Diferencias de Género. Revista Mexicana de Psicología, 32 (1), 27-36.

Nejad, M. K., Karami, A., Moazedian, A., \& Sedaghat, M. (2019). Compilation of the Structural Model of Psychological Well-Being Based on Optimism, Vitality and Positive Thinking With Mediation of Emotional Regulation. International Journal of Hospital Research, 8 (1), 1-10. 
Patki, S. M. (2016). Perceived social support and psychological well-being among teenagers: The role of gender and optimism. Indian Journal of Health and Wellbeing, 7 (7), 691694.

Pérez, E. M. (2017). Burnout y Optimismo en Psicología de la Actividad Física y el Deporte: un Estudio en Deportistas Universitarios. (Tesis de doctorado, Universidad de Murcia). Repositorio Institucional de la Universidad de Murcia. http://hdl.handle.net/10201/55470

Peterson, C., Seligman, M., \& Vaillant, G. (1988). Pessimistic explanatory style is a risk factor for physical illness: A thirty-five-year longitudinal study. Revista de personalidad y psicología social, 55 (1), 23-27.

Ramnath, C., \& Singh S. (2019). Psychological Well Being of Young Adults: The Role of Hope and Optimism. Journal of Xi'an University of Architecture \& Technology, 11 (12), 1104-1114.

Robles Ojeda, F. G., Galicia Moyeda, I. X., Sánchez Velasco, A., \& Pichardo Hernández, A. (2019). Estilos explicativos optimista-pesimista y depresión en estudiantes de bachillerato. Alternativas en Psicologia, 56 (43), 1-11.

Ryff, C. D. (1989). Happiness is everything, or is it? Explorations on the meaning of psychological well-being. Journal of Personality and Social Psychology, 57 (6), 10691081

Sánchez Hernández, O., \& Méndez Carillo, F. X. (2009). El Optimismo como Factor Protector de la Depresión Infantil y Adolescente. Clínica y Salud, 20 (3), 273-280.

Sandoval Barrientos, S., Dorner Paris, A., \& Veliz Burgos, A. (2017). Bienestar psicológico en estudiantes de carreras de la salud. Investigación en Educación Médica, 6 (24), 260266. https://doi.org/10.1016/j.riem.2017.01.004

Sarmiento, C., \& Fritz. E. (2018). Relación entre solución de problemas, estilo atribucional y afecto en universitarios de Huancayo. (Tesis de fin de grado, Universidad Peruana de Ciencias Aplicadas). Repositorio académico UPC. http://hdl.handle.net/10757/622691

Scheier, M. S., Carver, S. C., \& Bridges, M. W. (1994). Distinguishing optimism from neuroticism (and trait anxiety, self-mastery, and self-esteem): A reevaluation of the Life Orientation Test. Journal of Personality and Social Psychology, 67 (6), 1063-1068.

Wani, M. A. (2018). Depression, optimism-pessimism attitude and psychological well-being in adolescent boys and girls. Indian Journal of Health \& Wellbeing, 9 (5), 114-116.

World Health Organization. Constitution.WHO, 1948. 